\title{
Characterizing the thermally induced structural changes to intact porcine eye, part 1: second harmonic generation imaging of cornea stroma
}

Hsin-Yuan Tan

Chang Gung Memorial Hospital

Department of Ophthalmology

Linko 333, Taiwan

\section{Shu-Wen Teng}

Wen Lo

National Taiwan University

Department of Physics

Taipei 106, Taiwan

\section{Wei-Chou Lin}

National Taiwan University Hospital Department of Pathology

Taipei 100, Taiwan

\section{Sung-Jan Lin}

National Taiwan University Hospital

Department of Dermatology

Taipei 100, Taiwan

\section{Shiou-Hwa Jee}

National Taiwan University Hospital

Department of Dermatology

Taipei 100, Taiwan

and

National Taiwan University Hospital

Department of Dermatology

College of Medicine

Taipei 100, Taiwan

\section{Chen-Yuan Dong}

National Taiwan University

Department of Physics

Taipei 106, Taiwan

\begin{abstract}
We characterize the structural changes of porcine corneal structures from 25 to $90^{\circ} \mathrm{C}$ using second harmonic generation ( $\mathrm{SHG}$ ) microscopy. Our results show that porcine stroma undergoes several distinct stages of structural changes between 25 and $90^{\circ} \mathrm{C}$. A decrease in SHG intensity from 30 to $45^{\circ} \mathrm{C}$ and the existence of SHG intensity peaks at 53,65 , and $77^{\circ} \mathrm{C}$ correlate to distinct structural alterations of the corneal stroma. At higher temperatures, the SHG intensity decreases and a baseline in SHG signal is reached at $90^{\circ} \mathrm{C}$. Our results demonstrate that SHG microscopy is a useful technique for obtaining qualitative and quantitative information of thermally treated corneal fibers without histological or labeling procedures. With additional developments, SHG imaging may be developed into an effective imaging technique for in vivo characterization of cornea structural changes. () 2005 Society of Photo-Optical Instrumentation Engineers. [DOI: 10.1117/1.2012987]
\end{abstract}

Keywords: multiphoton microscopy; second harmonic generation; cornea; imaging.

Paper 04260RR received Dec. 30, 2004; revised manuscript received Apr. 24, 2005; accepted for publication Apr. 26, 2005; published online Oct. 10, 2005.

\section{Introduction}

The ability to monitor corneal structural changes is important in many ophthalmologic applications. Surgical procedures such as conductive keratoplasty and laser thermokeratoplasty depend on the structural alteration of corneal structure in achieving vision correction. ${ }^{1,2}$ Since the cornea is a highly transparent object, the development of an effective imaging technique to obtain both qualitative and quantitative information of corneal structural changes without histological or labeling procedures can be a useful technology in guiding surgery and other therapeutic procedures in ophthalmology. Presently, histological techniques remain the most commonly used imaging platform in characterizing corneal changes. ${ }^{3-6}$ However, the development of nonlinear optical microscopic techniques offers an exciting imaging modality capable of cir-

Address all correspondence to Dr. Chen-Yuan Dong. Tel: +886-2-3366-5155. Fax: +886-2-2363-9984. E-mail: cydong@phys.ntu.edu.tw cumventing the drawbacks of conventional histological procedures. While second harmonic generation (SHG) was used to image crystalline structures in the 1970 s, the biomedical applications of nonlinear optical microscopy flourished with the introduction of two-photon fluorescence microscopy pioneered by Webb's group in $1990 .^{7-9}$ There are a number of important advantages in the applications of nonlinear optical microscopy. First, since the excitation probability is proportional to the square of excitation intensity, sample excitation only occurs effectively at the focal point. Therefore, microscopic imaging with this technique results in images with excellent axial-depth discrimination without using confocal detection. In addition, since photodamage only occurs at the focal point, the overall specimen damage is greatly reduced. Finally, since the near-infrared laser sources used in multiphoton microscopy are absorbed and scattered less than the ultraviolet or visible photons used in one-photon excitation mi-

1083-3668/2005/10(5)/054019/5/\$22.00 @ 2005 SPIE 
croscopy, multiphoton imaging allows greater sample depths to be achieved without histological procedures. ${ }^{10}$

In harmonic generation imaging, the nonlinear polarization effect at a high electric field can be used to explore a sample's microscopic structures. In general, the polarization $P$ of a material can be expressed as

$$
P=\chi_{1} \otimes E+\chi_{2} \otimes E \otimes E+\chi_{3} \otimes E \otimes E \otimes E+\ldots,
$$

where $\chi_{1}, \chi_{2}$, and $\chi_{3}$ are, respectively, the first-, second-, and third-order susceptibility tensors, and $E$ and $\otimes$ represent the external electric field and tensor product operation, respectively. In a material with noncentrosymmetric symmetry, a nonvanishing second-order susceptibility tensor $\chi_{2}$ contributes to a second harmonic generation $(\mathrm{SGH})$ signal, where the angular frequency of the incident electric field frequency $\omega$ is converted into photons with frequency $2 \omega .{ }^{11} \mathrm{In}$ particular, it is known that the unique 3-D molecular structure of collagen 1 leads to SHG signal and that such a signal can lead to imaging of the cornea stroma. ${ }^{12-15}$ In this work, we demonstrate the application of SHG microscopy to obtain qualitative and quantitative information of thermal damage to cornea in intact porcine eyes. Our results show that SHG microscopy can be applied to characterize structural changes to the cornea without histological or labeling procedures.

\section{Methods}

\subsection{Second harmonic generation microscopy}

The experimental setup of our SHG microscope is similar to a multiphoton microscope previously described. ${ }^{11}$ In this experiment, the 880-nm output of a diode-pumped solid state (DPSS) laser (Millennia X, Spectra Physics, Mountain View, California) pumped, titanium-sapphire laser (Tsunami, Spectra Physics) was used as the SHG source. To control the laser power and circularize the laser output, a combination of a half-wave plate, polarizer, and quarter-wave plate was used. The circularly polarized laser is guided toward an $x-y$ scanner system (Model 6220, Cambridge Technology, Cambridge, Massachusetts), where angular deviations along the $x$ and $y$ axes are achieved. The scanned beam is directed into an upright microscope (E800, Nikon, Japan) using additional optics. The input of the microscope was modified to accommodate a pair of lenses for beam expansion. The 880-nm laser source is first beam expanded and then reflected toward the focusing, oil immersion objective (Plan Fluor, $20 \times$ NA 0.75) by a dichroic mirror (720DCSPXR, Chroma Technology, Brattleboro, Vermont). For the objective with NA of 0.75 , we can estimate the size of the focal spot from diffraction theory. The radial and axial spot sizes are $1.22 \lambda / \mathrm{NA}$ and $4 \lambda / \mathrm{NA}^{2}$ ( $\lambda$ is the laser wavelength used in focusing), respectively. ${ }^{16}$ For the wavelength of $880 \mathrm{~nm}$, the radial and axial focal sizes are estimated to be 1.4 and $6.3 \mu \mathrm{m}$. In this fashion, the angular deviation of the $x-y$ scanning system is translated to linear scanning of the focal spot across the cornea specimens. An average power of around $24 \mathrm{~mW}$ at the specimens was used for second harmonic signal generation. The SHG signal generated from the corneal collagen fibers was collected in the back-scattering geometry by the same focusing objective. After passing through the short-pass, dichroic mirror, an additional bandpass filter centered at $440 \mathrm{~nm}$ (HQ440/40,
Chroma Technology) was used to isolate the SHG signal. The photodetector used in these experiments was a single-photon counting photomultiplier tube (R7400P, Hamamatsu, Japan).

In this fashion, a SHG scan of the cornea specimen can be achieved. Each image we acquired is composed of 256 $\times 256$ pixels (pixel spacing $0.86 \mu \mathrm{m}$ ), and the measured intensity represents the detected SHG photons per pixel. To obtain both high resolution and large area imaging of the cornea specimens, an $x-y$ sample scanning stage (H101, Prior Scientific, United Kingdom) was used to translate the cornea specimen after each $256 \times 256$ pixel image was acquired. The movement of the sample scanning stage allowed the acquisition of partially overlapping SHG images from different positions of the specimen. Each large area scan was composed of a $6 \times 6$ array of the $256 \times 256$ pixel SHG images, and they are later assembled to generate a large area image 880 $\times 880 \mu \mathrm{m}^{2}$ in size.

\subsection{Porcine cornea}

The cornea specimens used in this experiment were freshly acquired from local markets at the earliest possible time after sacrifice. Intact porcine eyes were acquired from the markets at around $6 \mathrm{AM}$. The estimated sacrifice time of the pigs was around 3 AM. According to the vendor, the pigs were slaughtered at around 4 months of age using electric tools. Possible deterioration to the porcine eyes can lead to their opaqueness. However, the porcine eyes used in our experiments were examined to ensure optical clarity in the cornea. At each temperature, three sets of porcine eyes were used to ensure statistical significance in our results. As soon as the porcine eyes were brought back to the laboratory, extraocular muscles were removed and the eyes were immersed in PBS buffer at the ambient temperature. A temperature bath (B206, Firstek, Taiwan) was used to heat the PBS buffer in which the intact porcine eyes were immersed. The digital indicator of the temperature bath allowed the reading of the temperature reading to a precision of $\pm 0.1^{\circ} \mathrm{C}$. After the PBS bath reached the desired equilibrated temperature, the porcine eyeballs were placed into the heated buffer bath for $5 \mathrm{~min}$. From a previous study in measuring the thermo-mechanical properties of porcine cornea, the treatment time of $5 \mathrm{~min}$ is a reasonable estimate for the porcine cornea to reach thermal equilibrium. ${ }^{17}$ In this work, we present data that include 18 temperatures ranging from 25 to $90^{\circ} \mathrm{C}$. At the end of the thermal treatment cycle, the porcine eyes were removed and mounted inside an empty cover glass container filled with PBS buffer, covered with a standard number 1.5 cover glass, and mounted on the upright microscope for SHG imaging. By adjusting the relative distance between the objective and the cornea sample, the surface of the cornea can be found. Once a scanned SHG image of the corneal surface is acquired, sequential images between adjacent positions of the cornea can be acquired. The SHG images were acquired with the corneas still attached to the eyes, and no excision of the corneas was done. Since the natural state of the porcine cornea is a curved structure, we estimated that the SHG images obtained in this fashion were between 0 and $7 \mu \mathrm{m}$ from the corneal surface, and the measured SHG intensity represents an average value of the porcine cornea within this thickness. Since the porcine eyes were constantly placed in PBS buffer, they remain well hydrated. 
Tan et al.: Characterizing the thermally induced structural changes...
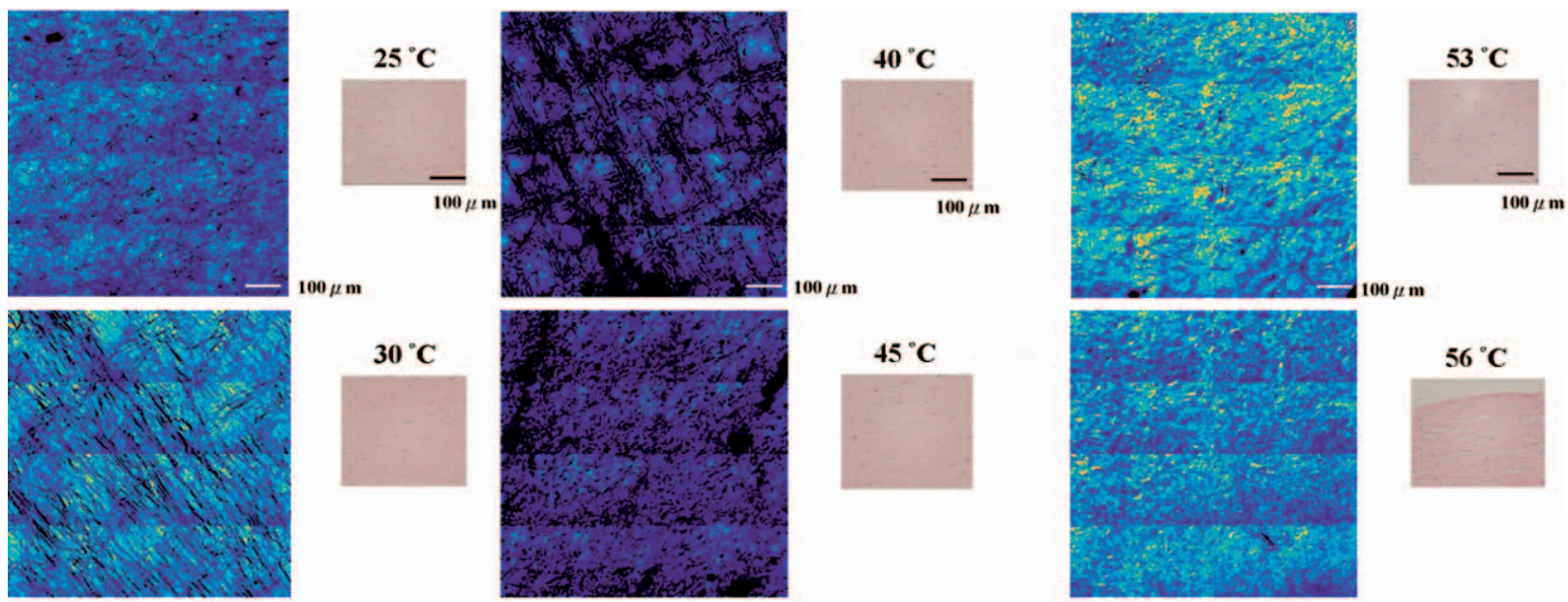

$100 \mu \mathrm{m}$
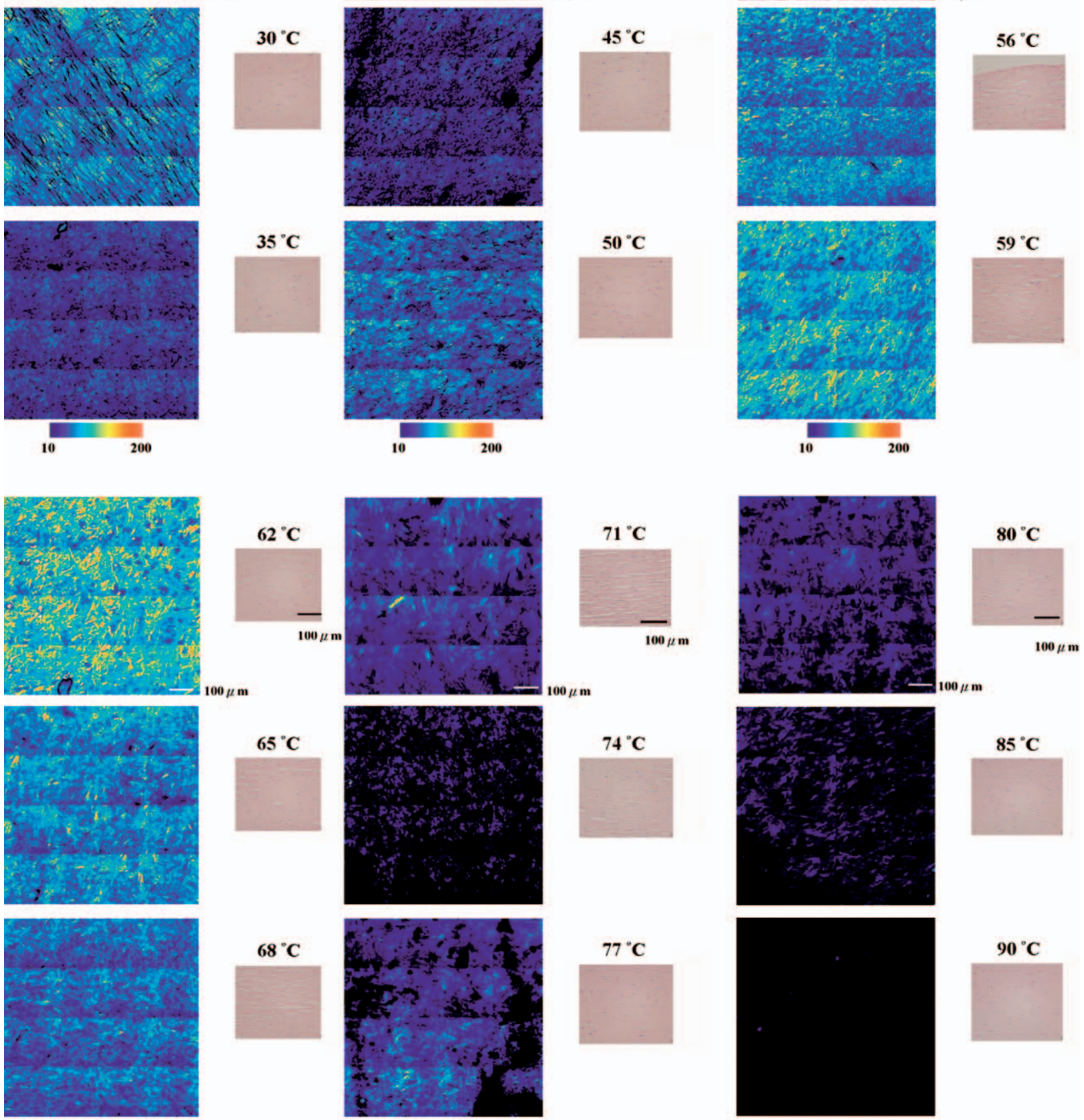

10

200
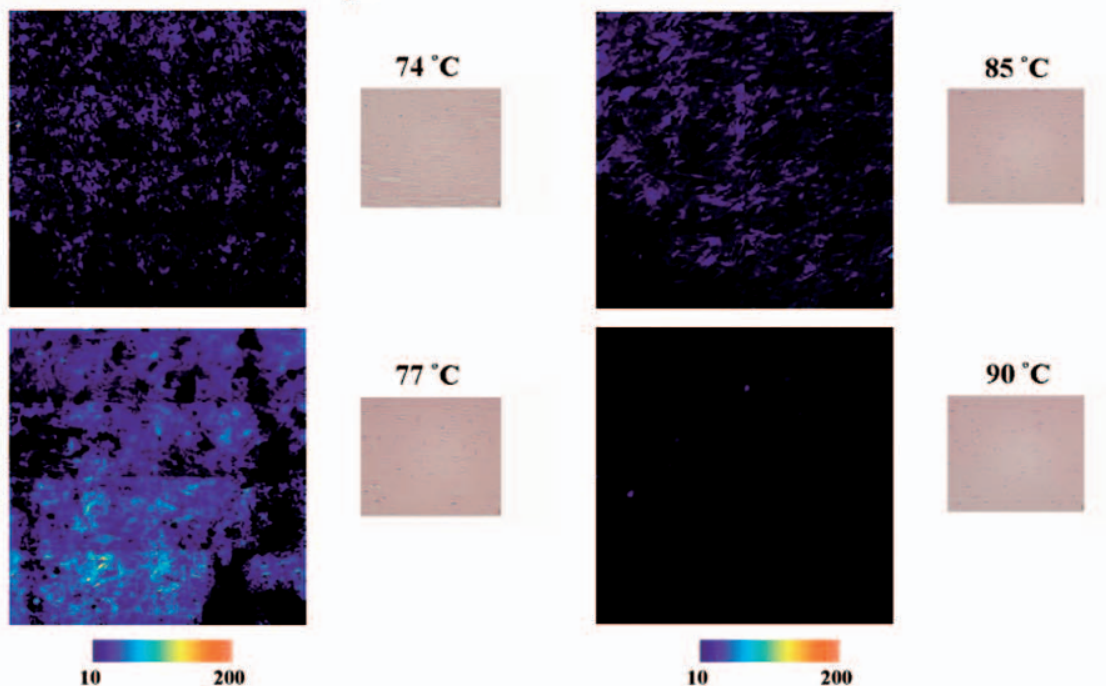

Fig. 1 Large area second harmonic images (left figures: $880 \times 880 \mu \mathrm{m}^{2}$ ) and HE images of porcine cornea after 5 min treatment at the respective temperatures. 
To acquire statistically significant results, at each temperature, three porcine eyes were used for SHG microscopic imaging. The analysis of the thermal effects on cornea collagen is completed in two ways. First, the large area SHG images are compared to the histological images. Furthermore, quantitative analysis is performed by averaging the SHG intensity across the large area scan. For each temperature, the results of the SHG intensity from three sets of porcine corneas were averaged and plotted.

After SHG imaging of the cornea specimens was completed, the samples were fixed in the formalin:PBS solution $(1: 9 \mathrm{v} / \mathrm{v})$ for histological processing. The processing of the porcine eye specimens was completed within $3 \mathrm{~h}$. Histological specimens of the thermally treated porcine corneas were prepared according to conventional histological procedures. The formalin:PBS stored porcine eyes were dehydrated and imbedded in paraffin. Thin sections with thicknesses around $5 \mu \mathrm{m}$ were prepared and labeled using hematoxylin and eosin (HE). The corneal collagen was labeled by eosin, which gives it the characteristic pinkish appearance. The histological images presented were obtained close to the corneal regions where the multiphoton imaging was performed.

\section{Results}

Using SHG microscopy, we acquired large area scans of the porcine corneas treated for $5 \mathrm{~min}$ at different temperatures from 25 to $90^{\circ} \mathrm{C}$. Shown in Fig. 1 are a representative set of the cross-sectional SHG images of the cornea stroma of 18 temperatures at $25,30,35,40,45,50,53,56,59,62,65,68$, $71,74,77,80,85$, and $90^{\circ} \mathrm{C}$. In each panel, the SHG images (left figures) are presented using the displayed color scale for indicating the minimum and maximum intensities used for display. For comparison, the corresponding HE images at each temperature are shown (right figures) for comparison.

In this manner, similar comparison between the SHG and HE images can be made. Visual examination of Fig. 1 reveals several important features. First, the collagen fibers associated with cornea stroma are clearly visible under SHG imaging. Furthermore, the SHG intensity changes as a function of temperature. Visual examination of the SHG images in Fig. 1 shows that with increasing temperature, distinct changes in SHG intensity occur. From 25 to $45^{\circ} \mathrm{C}$, SHG intensity appears to decrease with increasing temperature. However, starting at $50^{\circ} \mathrm{C}$, the SHG intensity increases and reaches maximum values between 50 and $71^{\circ} \mathrm{C}$. With additional increase in temperature, SHG intensity starts to decrease. However, an additional maximum in SHG signal is observed at around 74 to $77^{\circ} \mathrm{C}$. As the temperature continues to increase, the SHG signal decreases until $90^{\circ} \mathrm{C}$, where the SHG intensity has reached a baseline value. The corresponding histological images show that at temperatures less than $53^{\circ} \mathrm{C}$, the stroma collagen are organized into visible fibers. From 56 to $74^{\circ} \mathrm{C}$, regions of the cornea appear to have been grouped into forming separate domains without the finer fibrous structures visible at lower temperatures. The appearance of cavities is also apparent at these higher temperatures. At temperatures higher than $77^{\circ} \mathrm{C}$, the number of distinct domains decreases within the cornea. At $90^{\circ} \mathrm{C}$, the histological image shows that the corneal collagen has merged into a continuum distribution of mass with no discernible microscopic features. To add statistical signifi-

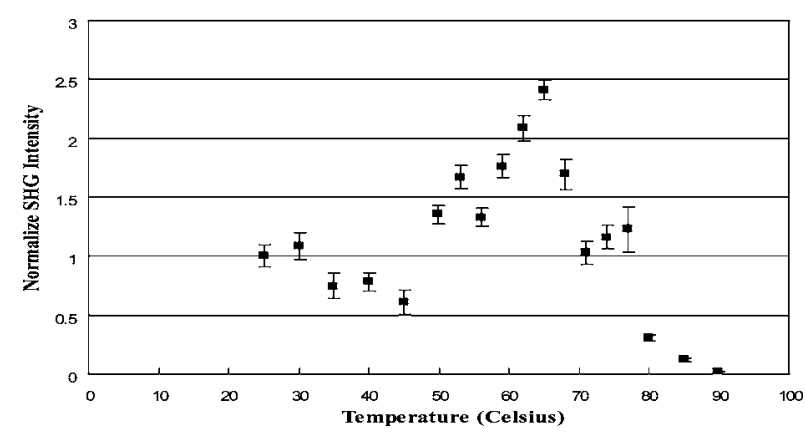

Fig. 2 Second harmonic intensity of porcine cornea stroma as a function of treatment temperature $(5 \mathrm{~min})$.

cance to our data, the intensity from the SHG images from two additional porcine eyes were measured and averaged. The statistical analysis of SHG intensity from the set of three porcine eyes at each temperature is shown in Fig. 2.

The plot in Fig. 2 shows that at 25 and $30^{\circ} \mathrm{C}$, the $\mathrm{SHG}$ intensities are essentially the same (within experimental error). In the higher temperature range between 35 and $45^{\circ} \mathrm{C}$, SHG intensity decreased. As the temperature further increases, the SHG intensity first increases to a peak at $53^{\circ} \mathrm{C}$ before reaching a main peak at $65^{\circ} \mathrm{C}$. With additional increase in temperature, the SHG signal first decreased and then increased to a secondary peak at around 74 to $77^{\circ} \mathrm{C}$. As the temperature continues to increase, the SHG intensity monotonically decreases to the background intensity at $90^{\circ} \mathrm{C}$. Note that these observations are difficult to confirm from the histological images alone. The sharp rise in SHG intensity at $65^{\circ} \mathrm{C}$ can be attributed to a sharp, irreversible structural change of the cornea stroma at that temperature.

\section{Discussion}

By applying SHG imaging to thermally treated cornea specimens in intact porcine eyes, we have shown that the collagen fibers of cornea stroma undergo four distinct structural changes in the temperature range between 25 to $90^{\circ} \mathrm{C}$. First, between 30 and $45^{\circ} \mathrm{C}$, the cornea fibrous structures remain largely intact. With additional increase in temperature, the corneal SHG intensity starts to increase and first reaches a peak value at $53^{\circ} \mathrm{C}$ before reaching a main peak at $65^{\circ} \mathrm{C}$. Histological examination of the cornea at $53^{\circ} \mathrm{C}$ shows that although the collagen fibers remain visible, they have grouped into domains separated by cavities. This phenomenon becomes even more apparent as the temperature increased. Finally, at $90^{\circ} \mathrm{C}$, the cavities are no longer visible and the cornea specimen seems to have merged into one continuous structure. These observations suggest that the SHG signal peaks observed at 53,65 , and 74 to $77^{\circ} \mathrm{C}$ correspond to the organization of collagen fibers into locally dense bundles made possible by the thermal disruption of cross-linking elements in the corneal stroma. The existence of multiple peaks suggests that the reorganization of corneal collagen occurs at three distinct phases between 53 and $77^{\circ} \mathrm{C}$, corresponding to different strengths of cross-linking within corneal collagen. The absence of organized histological structure and the lack of SHG signal at $90^{\circ} \mathrm{C}$ suggest that at sufficiently high temperatures, neither the cross-linking nor the hydrogen bonds 
Tan et al.: Characterizing the thermally induced structural changes...

within corneal stroma has the strength to maintain the noncentrosymmetric structure of collagen fibrils responsible for generating the SHG signal.

It is interesting to compare our results with the differential scanning calorimetry (DSC) data from porcine corneas published previously. It was shown that the porcine cornea starts to undergo thermal denaturation at $54.5^{\circ} \mathrm{C}$. At higher temperatures, porcine cornea absorbs more heat, reaching a maximum absorption temperature at $64.8^{\circ} \mathrm{C}$. As the temperature further increases, the slope in the DSC thermogram changes until a baseline value is reached at $81.8^{\circ} \mathrm{C}$. It was suggested that the slope changes in a DSC thermogram between 64.8 and $81.8^{\circ} \mathrm{C}$ correspond to the existence of subprocesses. ${ }^{17}$ Since we detected changes in SHG intensity starting at $30^{\circ} \mathrm{C}$, while the DSC thermogram did not reveal corresponding changes in heat flow, it seems that SHG imaging is a more sensitive technique capable of detecting more subtle changes in corneal structures. In addition, since the same primary maximum transition temperature was measured using both techniques (SHG $65^{\circ} \mathrm{C}$; DSC $64.8^{\circ} \mathrm{C}$ ), SHG imaging is able to detect the structural change measured using DSC. Furthermore, the temperature range where DSC data indicated the existence of subprocesses also corresponds well to the SHG intensity diagram. Specifically, the SHG intensity plot demonstrated a variation in slope and the existence of a secondary SHG peak at $77^{\circ} \mathrm{C}$. Despite the similarities of the SHG and DSC results, several differences do exist. First, the difference in baseline temperature $\left(\mathrm{SHG} 90^{\circ} \mathrm{C}\right.$; DSC $\left.81.8^{\circ} \mathrm{C}\right)$ may be interpreted by the fact that we used intact porcine eyes for SHG imaging, while small excised corneal specimens were used in DSC measurements. The additional mechanical support provided by the sclera in maintaining the corneal structure in intact eyes may alter the response of thermally treated corneal collagen. Secondly, SHG imaging reveals a local maximum at $53^{\circ} \mathrm{C}$. Such a change is missing in the DSC data, suggesting that the microscopic SHG imaging technique is more sensitive in revealing microscopic structural changes than the macroscopic heat flow measurement using DSC.

This work demonstrates that the nonlinear imaging modality of second harmonic generation microscopy may be used to obtain qualitative and quantitative information of collagen structural alterations in the cornea. Although SHG intensity is a far-field optical technique with spatial resolution in the micron range, the changes in SHG intensity are sensitive to alterations in cornea structures responsible for the second-order susceptibility tensor $\chi_{2}$ and other changes at the tissue level. In addition, since SHG microscopy depends on the intrinsic fiber orientations for signal generation, invasive histological or labeling procedures are not needed to obtain structural information valuable in guiding surgery and other therapeutic procedures of the cornea. Furthermore, since the SHG signal is a polarization effect rather than molecular excitation used in fluorescence microscopy, there is minimal photodamage to the specimens. Therefore, unlike other optical imaging techniques, SHG microscopy has the potential to be developed into an effective imaging technique for in vivo characterization of corneal structures. Our results show that SHG microscopy can be a valuable addition to the repertoire of imaging techniques in determining corneal structures, and may be developed into an effective imaging technology useful in guid- ing surgery and characterizing tissue engineered products intended for corneal repair.

\section{Acknowledgment}

The authors acknowledge the support from the National Research Program for Genomic Medicine, National Science Council, Taiwan (NSC 93-3112-B-002-033 and 94-3112-B002-015-Y) for this project

\section{References}

1. M. B. McDonald, P. S. Hersh, E. E. Manche, R. K. Maloney, J. Davidorf, and M. Sabry, "Conductive keratoplasty for the correction of low to moderate hyperopia: US clinical trial 1-year results on 355 eyes," Ophthalmology 109(11), 1978-1989 (2002).

2. R. Brinkmann, B. Radt, C. Flamm, J. Kampmeier, N. Koop and R. Birngruber, "Influence of temperature and time on thermally induced forces in corneal collagen and the effect on laser thermokeratoplasty," J. Cataract Refractive Surg. 26(5), 744-754 (2000).

3. M. N. AsiyoVogel, R. Brinkmann, H. Notbohm, R. Eggers, H. Lubatschowski, H. Laqua, and A. Vogel, "Histologic analysis of thermal effects of laser thermokeratoplasty and corneal ablation using Siriusred polarization microscopy," J. Cataract Refractive Surg. 23, 515526 (1997).

4. D. D. Koch, T. Kohnen, J. A. Anderson, P. S. Binder, M. N. Moore, R. F. Menefee, G. L. Valderamma, and M. J. Berry, "Histologic changes and wound healing response following 10-pulse noncontact holmium: YAG laser thermal keratoplasty," J. Refract. Surg. 12(5), 623-634 (1996).

5. T. Kohnen, S. E. Husain, and D. D. Koch, "Corneal topographic changes after noncontact holmium:YAG laser thermal keratoplasty to correct hyperopia," J. Cataract Refractive Surg. 22(4), 427-435 (1996).

6. T. Tanaka, S. Furutani-Miura, M. Nakamura, and T. Nishida, "Immunohistochemical study of localization of extracellular matrix after holmium YAG laser irradiation in rat cornea," Jpn. J. Ophthalmol. 44(5), 482-488 (2000).

7. R. Hellwarth and P. Christensen, "Nonlinear optical microscopic examination of structure in polycrystalline ZnSe," Opt. Commun. 12(3), 318-322 (1974).

8. J. N. Gannaway and C. J. R. Sheppard, "1978. Second-harmonic imaging in the scanning optical microscope," Opt. Quantum Electron. 10, 435-439 (1978).

9. W. Denk, J. H. Strickler, and W. W. Webb, "2-photon laser scanning fluorescence microscopy," Science 248(4951), 73-76 (1990).

10. P. T. C. So, C. Y. Dong, B. R. Masters, and K. M. Berland, "Twophoton excitation fluorescence microscopy," Annu. Rev. Biomed. Eng. 2, 399-429 (2000).

11. S. H. Lee, Y. Liu, H. C. Chen, L. L. Chiou, G. T. Huang, W. Lo, and C. Y. Dong, "Optical biopsy of liver fibrosis by use of multiphoton microscopy," Opt. Lett. 29(22), 2614-2616 (2004).

12. P. J. Campagnola and L. M. Lowe, "Second-harmonic imaging microscopy for visualizing biomolecular assay in cells, tissues and organisms," Nat. Biotechnol. 21(11), 1356-1360 (2003).

13. W. R. Zipfel, R. M. Williams, R. Christie, A. Y. Nikitin, B. T. Hyman, and W. W. Webb, "Live tissue intrinsic emission microscopy using multiphoton-excited native fluorescence and second harmonic generation," Proc. Natl. Acad. Sci. U.S.A. 100(12), 7075-7080 (2003).

14. A. Zoumi, A. Yeh, and B. J. Tromberg, "Imaging cells and extracellular matrix in vivo by using second-harmonic generation and twophoton excited fluorescence," Proc. Natl. Acad. Sci. U.S.A. 99(17), 11014-11019 (2002).

15. A. T. Yeh, N. Nassif, A. Zoumi, and B. J. Tromberg, "Selective corneal imaging using combined second-harmonic generation and two-photon excited fluorescence," Opt. Lett. 27(23), 2082-2084 (2002).

16. C. Y. Dong, P. T. C. So, C. Buehler, and E. Gratton, "Spatial resolution in pump-probe microscopy," Optik (Jena) 106, 7-14 (1997).

17. J. Kampmeier, B. Rodt, R. Birgruber, and R. Brinkmann, "Thermal and biomechanical parameters of porcine cornea," Cornea 19(3), 355-363 (2000). 